\title{
Segmentación y comportamiento de lealtad de los pacientes: un análisis clúster en los departamentos de salud de la Comunidad Valenciana*
}

\author{
Segmentation and Patient Loyalty: A Cluster Analysis of the Health Service Departments in the Valencian \\ Community
}

Segmentação e comportamento de lealdade dos pacientes: uma análise cluster nos departamentos de saúde da Comunidade Valenciana

\author{
Remedios Calero Gimeno ${ }^{\text {a }}$ \\ Gerencia de Atención Integrada de Hellín, España \\ ORCID: http://orcid.org/0000-0002-9847-847X \\ Martina G. Gallarza Granizo \\ Universitat de València, España \\ ORCID: http://orcid.org/0000-0003-2461-1247 \\ Sergio Gadea Peña \\ Conselleria de Sanidad de la Generalitat Valenciana, \\ España \\ ORCID: http://orcid.org/0000-0003-2769-2492
}

DOI: https://doi.org/10.11144/Javeriana.rgps17-34.sclp

Fecha de recepción: 10 Marzo 2017

Fecha de aceptación: 20 Octubre 2017

\section{Resumen:}

En un marco sanitario de fragmentación territorial y cohabitación de gestión pública con colaboración público-privada, tiene sentido analizar y clasificar los departamentos sanitarios, atendiendo a múltiples objetivos: conocer el comportamiento real de los pacientes cuando pueden elegir dónde ser atendidos, adaptar la cartera de servicios a las preferencias del paciente y organizar la asistencia sanitaria desde una óptica territorial, considerando si afecta el modelo de gestión. Se utilizan datos secundarios (más de dos millones) en la Comunidad Valenciana, con un análisis clúster del comportamiento de lealtad (captación, retención y deserción). Esta metodología permite la reagrupación por similitud de los departamentos, explicando su homogeneidad entre sí y heterogeneidad respecto a otros. Se muestra la bondad de este análisis en el sector sanitario. Asimismo, la clasificación obtenida supone una guía para la gestión estratégica de los servicios sanitarios (a nivel macro y micro) y ahonda en el debate de la colaboración público-privada.

Palabras clave: servicio de salud, comercialización de los servicios de salud, relaciones médico-paciente, administración hospitalaria, administración de los servicios de salud, hospitales.

\begin{abstract}
:
Under a health service framework marked by the territory segmentation and the coexistence of public administration and publicprivate ventures, it makes sense to analyze and classify the health service departments by considering the following objectives: to know the way patients actually behave when the choice of the service place is up to themselves; to adapt the service offer to the patient's preferences; and to organize health service based on the territory location, thus considering its influence on the management model. Secondary data from the Valencian Community (over two million data) are used in the cluster analysis of the patient loyal behavior (entering, retention and drop out). This methodology enables the department clustering based on their similarities, thus explaining the homogeneity and heterogeneity with each other. This analysis shows to be useful for the said health service sector. In addition, the resulting classification provides a guide for the strategic management of the health services (at the macro and micro levels) and goes deeper in the debate about public-private ventures.
\end{abstract}

Keywords: health service, health service marketing, doctor-patient relations, hospital administration, health service administration, hospitals. 


\section{Resumo:}

Em um marco sanitário de fragmentação territorial e coabitação de gestão pública com parceria público-privada faz sentido analisar e classificar os departamentos sanitários, atendendo objetivos múltiplos: conhecer o comportamento real dos pacientes quando puder eleger onde eles serem atendidos, adaptar o portfolio de serviços às preferencias do paciente e organizar a assistência sanitária desde una ótica territorial, considerando se afeta o modelo de gestão. Utilizam-se dados secundários (mais de dois milhões) na Comunidade Valenciana, com análise cluster do comportamento de lealdade (recrutamento, retenção e deserção). Essa metodologia permite a reagrupação por semelhança dos departamentos, explicando a sua homogeneidade entre se e a heterogeneidade em relação aos demais. Demonstra-se a bondade desta análise no setor sanitário. Da mesma forma, a classificação obtida supõe uma guia para a gestão estratégica dos serviços sanitários (no nível macro e micro) e aprofunda o debate da parceria público-privada.

Palavras-chave: serviço de saúde, comercialização dos serviços de saúde, relacionamentos médico-paciente, administração hospitalar, administração dos serviços de saúde, hospitais.

\section{Introducción}

La territorialidad forma parte de la idiosincrasia de multitud de sistemas nacionales de salud [1], pudiendo ser entendida como una estrategia de reorganización sanitaria [2]. Con el foco en el sistema sanitario español, el carácter territorial se plasma en una elevada descentralización $[3,4,5]$. Según lo establecido en la Ley General de Sanidad de 1986 [6] y las características del Estado de las Autonomías, el sistema sanitario público español es gestionado por las comunidades autónomas. A su vez, la ordenación sanitaria del territorio en cada comunidad autónoma se fundamenta en el artículo 56 de la misma Ley General de Sanidad de 1986, que establece las áreas de salud como las demarcaciones y estructuras básicas para la prestación sanitaria. A partir de esta legislación, cada comunidad autónoma ha definido territorialmente sus áreas de salud con su propia denominación (gerencias en Castilla La Mancha, departamentos en la Comunidad Valenciana, etcétera).

En el caso concreto de la Comunidad Valenciana, la región está ordenada en veinticuatro departamentos de salud, cinco de los cuales (véase tabla 1) son gestionados mediante una fórmula de colaboración públicoprivada - comúnmente llamada Modelo Alzira por ser esta la localidad donde se implantó el primer hospital gestionado bajo dicha fórmula en 1999-.

A su vez, cada departamento de salud cuenta con uno o dos hospitales y una red de centros de salud y consultorios auxiliares con los que ofrecer asistencia sanitaria al área geográfica asignada. Para comprender mejor la variedad y especificidad de estos departamentos, la tabla 1 explica la provincia en la que se ubica el departamento, el modelo de gestión y su tamaño, determinado por la Conselleria de Sanidad de la Comunidad Valenciana con base en la población asignada y la infraestructura hospitalaria (cartera de servicios y número de camas). 
TABLA 1

Clasificación de los departamentos de salud de la Comunidad Valenciana

\begin{tabular}{|c|c|c|c|c|}
\hline $\begin{array}{l}\text { Departamento de } \\
\text { Salud }\end{array}$ & Provincia & Tipo de gestión & Tamaño & $\begin{array}{l}\text { Población } \\
\text { protegida* }\end{array}$ \\
\hline Valencia La Fe & Valencia & Pública & Grande & 183244 \\
\hline General de Valencia & Valencia & Pública & Grande & 340393 \\
\hline Valencia Clínico & Valencia & Pública & Grande & 322800 \\
\hline Doctor Pesset & Valencia & Pública & Grande & 343865 \\
\hline Castellón & Castellón & Pública & Grande & 265626 \\
\hline Alicante & Alicante & Pública & Grande & 251244 \\
\hline Elche & Alicante & Pública & Grande & 152696 \\
\hline San Juan & Alicante & Pública & Grande & 200593 \\
\hline Gandia & Valencia & Pública & Mediano & 172157 \\
\hline Villajoyosa & Alicante & Pública & Mediano & 163716 \\
\hline Orihuela & Alicante & Pública & Mediano & 158042 \\
\hline Elda & Alicante & Pública & Mediano & 187037 \\
\hline Alcoy & Alicante & Pública & Mediano & 134714 \\
\hline Xàtiva-Ontinyent & Valencia & Pública & Mediano & 193854 \\
\hline Amau de Vilanova & Valencia & Pública & Mediano & 291364 \\
\hline Sagunto & Valencia & Pública & Mediano & 144619 \\
\hline La Plana & Castellón & Pública & Mediano & 181426 \\
\hline La Ribera & Valencia & $\begin{array}{l}\text { Colaboración público- } \\
\text { privada }\end{array}$ & Mediano & 251475 \\
\hline Denia & Alicante & $\begin{array}{l}\text { Colaboración público- } \\
\text { privada }\end{array}$ & Mediano & 152769 \\
\hline Torrevieja & Alicante & $\begin{array}{l}\text { Colaboración público- } \\
\text { privada }\end{array}$ & Mediano & 162407 \\
\hline Manises & Valencia & $\begin{array}{l}\text { Colaboración público- } \\
\text { privada }\end{array}$ & Mediano & 193417 \\
\hline Elx-Crevillent & Alicante & $\begin{array}{l}\text { Colaboración público- } \\
\text { privada }\end{array}$ & Mediano & 148249 \\
\hline Vinaroz & Castellón & Pública & Pequeño & 84596 \\
\hline Requena & Valencia & Pública & Pequeño & 48079 \\
\hline
\end{tabular}

Fuente: datos recogidos en el Sistema Información Poblacional (SIP) de la Generalitat Valenciana.

* Media 2012.

Esta fragmentación no evita que el paciente se mueva dentro del territorio de la Comunidad Valenciana en aras de obtener asistencia sanitaria. Es más, se reconoce la libre elección de médico y centro sanitario en esta comunidad autónoma [7]. La libre elección [8], además de permitir al paciente tomar decisiones sobre su propia salud, es una muestra de la relación médico-paciente existente. Es decir, la libre elección supone la manifestación de las preferencias de los pacientes. Este hecho conlleva una doble utilidad: para los pacientes, por un lado, permite otorgar un voto de confianza al profesional sanitario, valorando su trabajo y su profesionalidad; y por otro, para los gestores, permite conocer las preferencias de los pacientes y predecir su comportamiento a efectos de reorganización del servicio. 
Todo ello adquiere una relevancia aún mayor si consideramos que el servicio sanitario se caracteriza por la especial relación médico-paciente [9], en la que el trato no tiene lugar entre iguales (relación de agencia), sino que el paciente acude buscando consejo médico en una situación de incertidumbre y cierta vulnerabilidad. En estas circunstancias, se establece un vínculo entre ambos actores que puede generar una gran confianza del paciente en su médico $[10,11]$. A su vez, la confianza alcanzada podría traducirse en un comportamiento de lealtad del paciente. De hecho, la lealtad cuenta con una base afectiva, entendida como un vínculo emocional en el que se experimenta un deseo de asociarse y dar apoyo a alguien o a algo [12]. En este sentido, encontramos una vinculación directa entre la relación de los pacientes con los profesionales y la lealtad manifestada por los primeros.

Más allá de esta relación médico-paciente, el servicio sanitario se caracteriza también por la relevancia de los paraservicios [9], ya que el paciente ante su dificultad para evaluar si el servicio recibido ha sido el adecuado, otorga una especial valoración a otros atributos del entorno que envuelve al servicio, tanto tangibles - ej. equipamiento, confort - $[13,14,15]$ como intangibles - ej. profesionalidad, rapidez- [16]. De este modo, el paciente no sería atraído por el centro sanitario con base en el vínculo establecido con el profesional, sino que podría verse potenciado este efecto con el ofrecimiento de cada centro de paraservicios de alta valoración para el usuario.

Por todo ello, y yendo un paso más allá si consideramos sistemas de financiación capitativa y facturación intercentros (en los que los movimientos de pacientes conllevan un aumento o detracción del presupuesto de un hospital), como es el caso de la Comunidad Valenciana, resulta de gran relevancia conocer en qué medida logran los diferentes departamentos de salud obtener la lealtad de sus pacientes.

A fin de estudiar la lealtad alcanzada, el análisis toma en consideración la observación de los movimientos de los pacientes entre departamentos de salud. Estos movimientos entre departamentos se plasman en la figura 1, interpretados a la luz de tres variables estratégicas de la lealtad: captación, retención y deserción. A este respecto, la captación se entiende como el establecimiento de relaciones con nuevos pacientes $[17,18]$, la retención se define como el comportamiento reiterativo del paciente en acudir a recibir asistencia [19, 20], mientras que la deserción tiene lugar cuando se materializa la ruptura de la relación por parte del paciente [21]. 


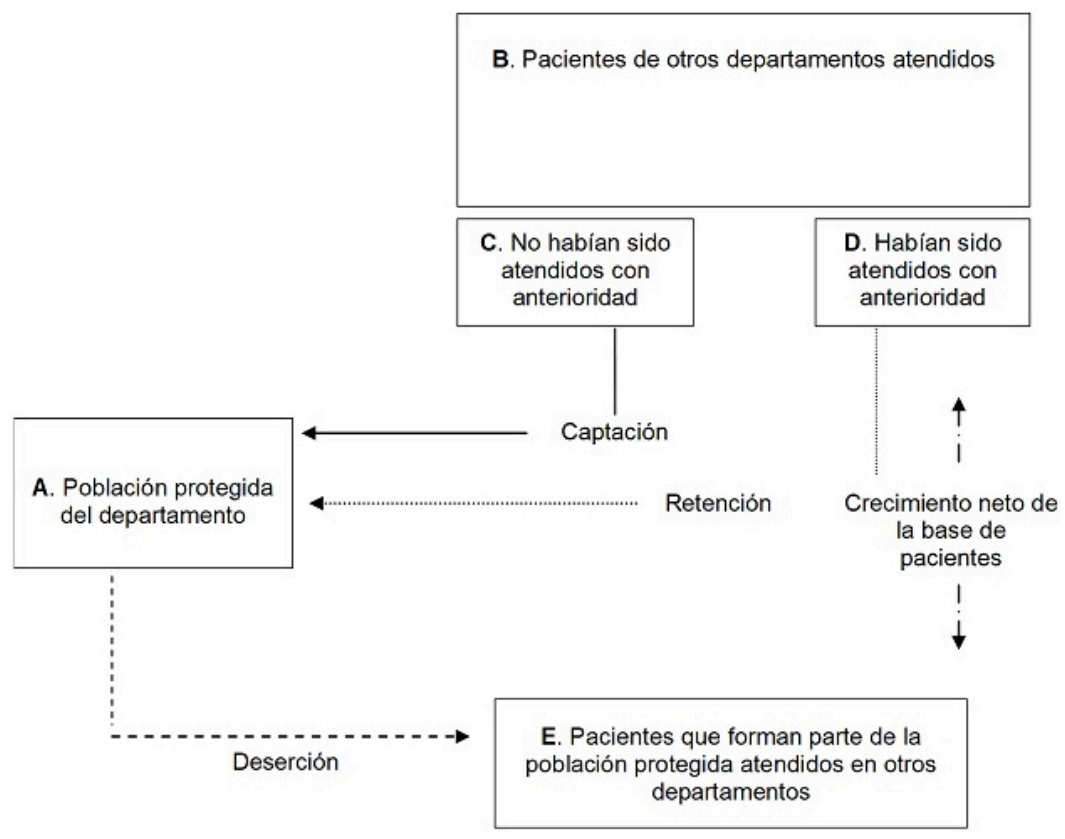

FIGURA 1

Captación, retención y deserción de pacientes entre departamentos de salud de la Comunidad Valenciana Fuente: adaptación de Calero y Gallarza [22].

Cada departamento de salud cuenta con una población protegida asignada (A). Asimismo, puede atender a pacientes de otros departamentos de salud (B) que habrán sido tratados con anterioridad (C) o no (D). Por otro lado, parte de la población protegida del departamento acudirá a otros departamentos de salud a recibir asistencia sanitaria (E).

En este marco, entendemos la captación (trazo continuo) como la proporción de pacientes de otros departamentos de salud que, sin haber sido tratados con anterioridad, son atendidos en un departamento, en relación con la población protegida de este departamento. Por retención (trazo punteado), consideramos la proporción de pacientes de otros departamentos de salud que, habiendo sido tratados con anterioridad, son atendidos, respecto de la población protegida de este departamento. Por su parte, entendemos por deserción (trazo discontinuo) la proporción de pacientes de la población protegida que son atendidos en un departamento de salud al que no estaban asignados. Para hacer estas variables operativas en nuestro estudio, estos parámetros se calculan como una proporción respecto a la población asignada, para así minimizar el sesgo que supondría el tamaño del hospital, ya que la dotación y la infraestructura normalmente suelen ajustarse a la población protegida con que cuenta cada departamento.

La metodología empleada para la consecución del trabajo consiste en un análisis que determinará qué grupos de departamentos cuentan con una mayor homogeneidad en el comportamiento del paciente y cuáles plantean mayor heterogeneidad respecto al resto de grupos. Dada además la particularidad de la cohabitación de departamentos públicos y de colaboración público-privada en la Comunidad Valenciana, como un objetivo secundario del trabajo, el estudio hace hincapié en los grupos en los que se sitúan los departamentos gestionados mediante el modelo Alzira. Asimismo, se demuestra cómo el análisis clúster puede ser usado en este tipo de estudios en el ámbito de la sanidad, con gran relevancia de cara a la gestión sanitaria.

Con todo ello se adoptará una triple perspectiva: de demanda, analizando el comportamiento manifestado por los pacientes; de oferta, considerando la gestión de los departamentos de salud; y a nivel de macrogestión, 
en cuanto a la visualización del mapa sanitario por agrupaciones, facilitando con ello la adopción de políticas sanitarias.

\section{Material y métodos}

\section{Justificación y pautas metodológicas seguidas para el análisis de grupos}

Con este marco de referencia, a efectos de observar el comportamiento del paciente en un ámbito de segmentación territorial, empleamos un análisis de grupos. El análisis de grupos constituye una herramienta de análisis de datos de gran utilidad en multitud de situaciones diferentes, que resulta de aplicabilidad en ámbitos de investigación tales como psicología, biología, sociología, ingeniería o economía, entre otros [22, 23]. En el campo del marketing, observamos su aplicación en la clasificación de objetos, la determinación de estructuras de mercado, el análisis de semejanzas y diferencias entre nuevos productos, el diseño de campañas promocionales, o la evaluación del desempeño de las empresas, de tal manera que logra identificar agrupaciones desde una óptica estratégica $[22,24,25,26]$. Así, encontramos trabajos aplicados tanto a empresas -e. g. para agrupar diferentes empresas en función de su enfoque al entorno [27] - como a organizaciones - e. g. aplicado, desde una aproximación socioeconómica y demográfica, a los departamentos de la provincia de Córdoba en Argentina [28] —. En el caso de España, este análisis se ha aplicado con anterioridad al sector sanitario, concretamente a hospitales: por ejemplo, en un estudio [29], mediante un análisis de grupos, se caracteriza y diferencia los hospitales de la Comunidad Valenciana en lo relativo a producción de servicios y consumo de recursos sanitarios y económicos. También se emplea [30] esta metodología para definir grupos homogéneos de hospitales del Sistema Nacional de Salud español que permitan comparar indicadores de funcionamiento y costes, o bien para distinguir grupos de hospitales atendiendo a su capacidad para proveer cuidados a las personas mayores [31].

Metodológicamente, se ha empleado una fuente secundaria, la base de datos interna de la Conselleria de Sanidad de la Comunidad Valenciana denominada COMPAS [32]. En dicho aplicativo informático COMPAS se recogen los datos de flujos de pacientes y asistencias entre departamentos. En el presente estudio se han analizado más de dos millones de asistencias que tuvieron lugar en un año natural, el 2012. Los registros estudiados corresponden al ámbito de la atención especializada, y más concretamente a la hospitalización y la cirugía sin ingreso. A fin de estudiar el comportamiento del paciente basado en la libre elección de centro sanitario, se han descartado las atenciones que tienen que ser realizadas en otros departamentos de salud, debido a que el departamento al que pertenece el paciente no cuenta con este servicio, y se han obviado las asistencias realizadas a pacientes de otras comunidades autónomas y países por no tener departamento asignado en la Comunidad Valenciana. Los datos se han analizado con el programa Statistical Package for Social Sciences (SPSS) versión 15.0.

En el presente trabajo, se ha aplicado un análisis clúster, definido [33] como "un método estadístico multivariable de clasificación de datos" (p. 269). Como es sabido, este método de agrupamiento se basa en la identificación de grupos de casos con características comunes, pero distintas a las de otros grupos [33, 34, 35]. Su utilidad reside en que permite: describir una taxonomía, simplificar los datos mediante la identificación de una estructura y determinar la relación entre las observaciones $[23,36]$.

De manera previa a la exposición del análisis realizado, creemos necesario reseñar las pautas metodológicas seguidas en nuestro análisis de grupos, con el fin de facilitar su interpretación:

- De entre los diferentes procedimientos, entendemos como más apropiado el análisis de conglomerados jerárquico. Se trata de "una técnica aglomerativa en la que, partiendo de los elementos individualmente considerados, se van creando grupos hasta llegar a la formación de un único grupo constituido por todos los elementos" [37]. 
- Aplicado a nuestra investigación, seleccionamos dos variables: las tasas de captación y retención consideradas de manera conjunta, y la tasa de deserción de pacientes; y como casos, los valores de estas variables en cada uno de los veinticuatro departamentos de salud de la Comunidad Valenciana. La consideración de estas dos variables nos permite distinguir para cada departamento de salud, por una parte, su capacidad de establecer y mantener relaciones con los pacientes de otros departamentos (captación y retención) y, por otra, la medida en que mantiene la relación con los pacientes que tiene inicialmente asignados (deserción).

- El método de conglomeración empleado es la vinculación intergrupo, aplicando como medida del intervalo la distancia euclídea. El método de vinculación intergrupo, también conocido como vinculación promedio, calcula la distancia entre dos grupos como la distancia promedio existente entre todos los pares de elementos de ambos conglomerados. De este modo, permite aprovechar la información de todos los miembros de los grupos que se comparan. En cuanto a la distancia euclídea, se trata de la medida de disimilaridad usada por defecto para datos de intervalo $[35,37,38]$.

\section{Resultados}

\section{Reagrupación por similitud de los departamentos de salud de la Comunidad Valenciana: proceso de fusión de departamentos}

El procedimiento de análisis de grupos jerárquico empleado nos va a permitir agrupar los veinticuatro departamentos de manera progresiva hasta formar un único grupo o conglomerado, siendo de tanta importancia los grupos resultantes como la observación de las distancias entre elementos a fin de determinar la homogeneidad entre estos últimos [26]. Así, consideramos relevante plasmar el proceso de formación de conglomerados donde puede apreciarse tanto la distancia existente entre los elementos analizados como la secuencia de agrupación progresiva de los mismos. Primeramente, mostramos la matriz de distancias entre los Departamentos de Salud (véase tabla 2). Esta matriz recoge las disimilaridades entendidas como el grado de lejanía entre los elementos analizados. En esta visión, los valores más altos indican una mayor lejanía; mientras que, si los casos se encuentran juntos, la distancia es nula. Como podemos apreciar, la diagonal contiene el valor cero al relacionarse cada Departamento consigo mismo.

TABLA 2

Matriz de distancias entre departamentos de salud

\begin{tabular}{|c|c|c|c|c|c|c|c|c|c|c|c|c|c|c|c|c|c|c|c|c|c|c|c|c|}
\hline epartamento & vinaroz & Castellon & La Plana & Sagunto & Clinico & Anau & $\mathrm{LaFe}$ & Requena & $\begin{array}{c}\begin{array}{c}\text { Valencia } \\
\text { Gral }\end{array} \\
\text { a }\end{array}$ & \begin{tabular}{|c|} 
Dr. \\
Peset \\
\end{tabular} & \begin{tabular}{|c|c|}
$\begin{array}{c}\text { Le } \\
\text { Ribera }\end{array}$ \\
\end{tabular} & Gandia & Denia & \begin{tabular}{|c|} 
Xattiva- \\
Ont
\end{tabular} & Alcoy & Villajoyosa & $\begin{array}{l}\text { San } \\
\text { Juan }\end{array}$ & Elda & Aicante & Elche & Crihuela & Torrevieja & Manises & \begin{tabular}{|l|} 
Ello \\
Creve \\
\end{tabular} \\
\hline Winaroz & & $\overline{B 4}$ & 9.7 & & 1880 & 205.0 & 36485 & & & 1906 & 18.5 & 8.2 & 1.1 & 58 & 0.8 & 1.2 & 49.1 & 0.1 & 83.7 & 152 & 0.8 & 2.6 & 98.9 & \\
\hline & 8.4 & 0.0 & 18.9 & 3 & 180.5 & 238.9 & & & 125.7 & & 7.5 & & 5.7 & 16.8 & & 5.4 & 57.6 & 8.6 & 43.0 & 9.1 & 9.7 & 5.1 & & \\
\hline & 9.7 & 189 & 0.0 & 2.7 & 114.8 & 128.7 & 34929 & 17.5 & 93.7 & 114.9 & 11.3 & 0.6 & 13.8 & 0.7 & 54 & 15.9 & 15.5 & 9.9 & 74.2 & 7.7 & 5,1 & 19.8 & 47.9 & \\
\hline & 20.0 & 28.3 & 2.7 & 0.0 & 90.8 & 107.3 & 3419.5 & 7.1 & 78.4 & 93.1 & 12.2 & 3.6 & 23.8 & 5.1 & 14.1 & 27.3 & 7.0 & 20.2 & 73.0 & 8.4 & 13.1 & 31.8 & 30. & 4.2 \\
\hline & 18a.0 & 180.5 & 114.8 & 90.8 & 0.0 & 1.0 & 71.7 & 62.2 & 17.2 & 23.5 & 119.7 & \begin{tabular}{|l|}
125.8 \\
\end{tabular} & 197.6 & 1326 & 169.7 & 205.9 & 49.7 & 186.4 & 132.4 & 111.8 & 168.0 & 2160 & 24.3 & 128.5 \\
\hline & 205.0 & 9 & $12 B, 7$ & 107.3 & 51.0 & 0.0 & 32.9 & a & 100.9 & 5.5 & 179.4 & 131.9 & 224.8 & 142.5 & $18 \mathrm{C}$ & 3.7 & 64.6 & 200.1 & 269.0 & 164.1 & 181.6 & & 39.1 & 153. \\
\hline & 384 & & & 3419.5 & 2771.7 & 3532.9 & 00 & 3204.7 & 2576.4 & 3269.7 & 3203.3 & \begin{tabular}{|l|} 
\\
3573.6 \\
\end{tabular} & $\begin{array}{r}2840 \\
3808.6\end{array}$ & $\begin{array}{l}1420 \\
3564.4\end{array}$ & $\begin{array}{l}100 \\
3647 \\
\end{array}$ & & 32982 & \begin{tabular}{|l|}
2612.6 \\
610
\end{tabular} & 2634.8 & 32342 & 3808.3 & & 3191.3 & 3448 \\
\hline & & & & 7.1 & 62.2 & $\begin{array}{r}5002.9 \\
98.2\end{array}$ & 32047 & 0.0 & 55.0 & $\begin{array}{r}309.9 \\
789\end{array}$ & $\begin{array}{r}303.9 \\
\end{array}$ & $\begin{array}{r}300.0 \\
20.2\end{array}$ & 48.5 & $\begin{array}{r}5004.4 \\
23.1\end{array} \mid$ & 5041 & & $\begin{array}{r}32502 \\
60\end{array}$ & [501201 & $\begin{array}{r}2004.6 \\
62.3\end{array}$ & 32344 & 3000.9 & & 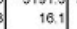 & $\mid$ \\
\hline & & $\begin{array}{l}425.7 \\
12.7\end{array}$ & 93 & 14 & & $\begin{array}{r}908.9 \\
1089\end{array}$ & & 55.0 & 0.0 & 67.5 & 80.5 & 7.1 & \begin{tabular}{|l|}
450.1 \\
155.1
\end{tabular} & $\begin{array}{r}209.5 \\
\end{array}$ & 137 & & 503 & & 64.3 & & 133 & & 429 & 100 \\
\hline & & & & & & & & & 5 & 0.0 & 51.4 & .9 & & 0.1 & 167 & & 4 & & T & & 167.6 & & & 13 \\
\hline & 16 & & 17.3 & & & & & & & & 0.0 & & 6 & 12.8 & & & & & 31.4 & & 13.3 & & & \\
\hline & & & 0.6 & 3.6 & & & & & 7.1 & 99 & 1 & & 14.0 .0 & 12 & & 14 & 18 & & & & & 4 & & \\
\hline & & & & 23.6 & & & & & & & & 3 & & 9 & & & & & & & & 0.7 & 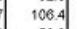 & \\
\hline & & & 0.7 & 5.1 & & & & & .5 & & 12.8 & 0.3 & & 0 & & 11.2 & 21. & & 80 & & & 14.8 & 58.3 & \\
\hline & & & & 1 & & & & & & 167.8 & & 4. & & 2 & & & & & & & & & 84.5 & \\
\hline & & 5. & & 27.3 & & & & & 180.3 & 21 & & & & & & & 60 & & & & & & 14.0 & \\
\hline & & & & & & & & & & & & 8.4 & 55.6 & 21.8 & & & & & & & & & & \\
\hline & & 6.6 & 9.9 & & & 208 & & & $146.0 \mid$ & & & & 0.8 & & & & & & & & & & & \\
\hline & & 430 & ( & 73.0 & & & & & & & & & 76 & 80 & & & & & & & & & & \\
\hline & 15 & & & & & & & & & & & 10.6 & 14. & & & 16 & & & & & & & & \\
\hline & 0 & & & 13 & & & & & & & & & & & & & & & & & & & & \\
\hline & & & & & & & & & 108.2 & & & & & & & & & & & & & & & \\
\hline & & & & & & & & & & & & & & & & & & & & & & & & \\
\hline & & & & & & 33.5 & 400.4 & & 100. & & & & & & 5.0 & & 21.3 & & 62.5 & 3.7 & 4.4 & 13.5 & 54.4 & \\
\hline
\end{tabular}

Fuente: elaboración propia.

La lectura de la tabla 2 nos muestra, en primer lugar, cómo La Fe se sitúa a la mayor distancia del resto de departamentos. En segundo lugar, los departamentos Valencia-Clínico, Valencia-Arnau, General de Valencia, 
Doctor Peset y Alicante cuentan con distancias que, sin llegar a los valores de La Fe, son de dos dígitos respecto de la mayoría de los departamentos. En tercer lugar, observamos cómo las distancias del resto de los departamentos son reducidas, los valores más elevados se presentan al calcular su lejanía respecto de los departamentos que acabamos de mencionar.

En la tabla 2, con base en las distancias mostradas en la tabla 1, se describe el proceso de agrupación de los departamentos, recogiendo el historial de conglomeración: del análisis de los veinticuatro departamentos de salud se obtiene una agrupación en veintitrés etapas en la que se van fusionando dos elementos por etapa. 
TABLA 3

Historial de conglomeración

\begin{tabular}{|c|c|c|c|c|c|c|}
\hline \multirow[t]{2}{*}{ Etapa } & \multicolumn{2}{|c|}{ Coeplonrrado que se combina } & \multirow[t]{2}{*}{ Coeficintes } & \multicolumn{2}{|c|}{$\begin{array}{l}\text { Elapa en la que el conplotierado } \\
\text { aperece por primera vez }\end{array}$} & \multirow[t]{2}{*}{ Prớcina cteps: } \\
\hline & Cond 1 & Cond 2 & & $\operatorname{Con} 1.1$ & Congl 2 & \\
\hline 1 & Dena & Villioyosa & 0.369 & 0 & 0 & 5 \\
\hline 2 & Vmeroz & Eks & 0.433 & 0 & 0 & 8 \\
\hline 3 & Groxdia & Xitwa-Oat. & 0,472 & 0 & 0 & 7 \\
\hline 4 & Akoy & Onżeb & 0.516 & 0 & 0 & 8 \\
\hline 5 & Deria-Virijoycosa & Tonevizja & 0.661 & 1 & 0 & 9 \\
\hline 6 & La Rbera & Elde & 0.728 & 0 & 0 & 13 \\
\hline 7 & La Phan & Gandia-Xinka-Onz. & 0.893 & 0 & 3 & 10 \\
\hline 8 & VinarozEda & Alooy-Oninela & 0.985 & 2 & 4 & 9 \\
\hline 9 & $\begin{array}{c}\text { Vinvoz-Ela-Aboy" } \\
\text { Orlacla }\end{array}$ & $\begin{array}{l}\text { Deria-Vilyoyosar } \\
\text { Torrevicja }\end{array}$ & 1.575 & 8 & 5 & 15 \\
\hline 10 & $\begin{array}{l}\text { La Plem-Grodin-Xieva- } \\
\text { One. }\end{array}$ & Elx-Crev. & 1.782 & 7 & 0 & 11 \\
\hline 11 & $\begin{array}{c}\text { La Plasn-Ganlin-Xítra: } \\
\text { Ont-Ex-Crey. }\end{array}$ & Saputo & 2.130 & 10 & 0 & 15 \\
\hline 12 & Renuem & San ham & 2.285 & 0 & 0 & 16 \\
\hline 13 & Castelion & La Ruera-Bdie & 3.017 & 0 & 6 & 18 \\
\hline 14 & Ammas & D. Pesert & 3.023 & 0 & 0 & 20 \\
\hline 15 & $\begin{array}{c}\text { Vineroz-EXla-Akoy- } \\
\text { Onluxh- Denis-Vilyjoyosa- } \\
\text { Tonevicja }\end{array}$ & $\begin{array}{l}\text { La Phim-Gandia- } \\
\text { Xatha-Oat-Ek- } \\
\text { Crev-Sagno }\end{array}$ & 3.428 & 9 & 11 & 18 \\
\hline 16 & Requero-San Jua & Mraises & 3.570 & 12 & 0 & 19 \\
\hline 17 & Clinko & Vakncia Gral. & 3.945 & 0 & 0 & 20 \\
\hline 18 & 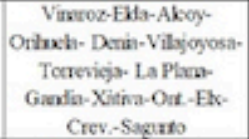 & $\begin{array}{c}\text { Castebio-La Ribera. } \\
\text { Eche }\end{array}$ & 4.421 & 15 & 13 & 19 \\
\hline 19 & 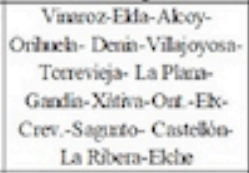 & $\begin{array}{c}\text { Requem-Sin Jabr } \\
\text { Mrises }\end{array}$ & 7.167 & 18 & 16 & 21 \\
\hline 20 & Clinico-Valercia Gral & AmaubDr. Peset & 9.581 & 17 & 14 & 22 \\
\hline 21 & 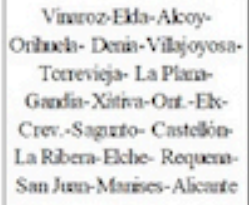 & Alicante & 11.015 & 19 & 0 & 22 \\
\hline 22 & 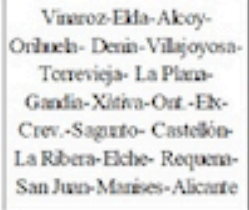 & $\begin{array}{c}\text { Clrico-Valencia Grat- } \\
\text { Amsu-Dr.Peset }\end{array}$ & 12.394 & 21 & 20 & 23 \\
\hline 23 & 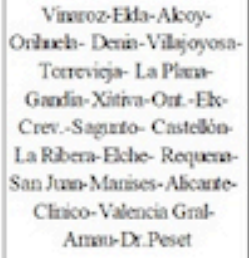 & LaFe & 79.094 & 22 & 0 & 0 \\
\hline
\end{tabular}

Fuente: elaboración propia. 
La columna conglomerado que se combina indica los dos elementos fusionados en la etapa, ya sean departamentos o grupos formados previamente. Los dos primeros departamentos en fusionarse son Denia y Villajoyosa, debido a que cuentan con la menor distancia entre ellos (coeficiente). En una segunda etapa se fusionan Vinaroz y Elda. En la tercera, Gandía y Xàtiva-Ontinyent se unen, mientras que en la cuarta lo hacen Alcoy y Orihuela. Es en la quinta etapa donde aparece el primer departamento, Torrevieja, que se fusiona con un grupo creado previamente: Denia-Villajoyosa. A partir de esta etapa, se van uniendo departamentos a grupos, departamentos entre sí y también grupos entre grupos. Es de destacar cómo Alicante no se incorpora a un conglomerado hasta la etapa número 21. Del mismo modo, La Fe es el último departamento en fusionarse, haciéndolo en la etapa final. Este hecho responde a la gran distancia a la que se sitúan Alicante y La Fe respecto del resto de los departamentos. Como puede observarse, las distancias de fusión (coeficientes) aumentan progresivamente conforme avanzan las etapas de conglomeración. El salto más importante tiene lugar en la última etapa donde el coeficiente pasa de 12.394 a 79.094. Precisamente, es en esta etapa donde se incorpora el último departamento, $\mathrm{La} \mathrm{Fe}$, al grupo, lo que denota que se trata de un valor extremo y pone en evidencia un comportamiento de los pacientes en este departamento sustancialmente diferente al manifestado en el resto de los departamentos de salud de la Comunidad Valenciana.

\section{Grupos de departamentos de salud en función del comportamiento de lealtad de los pacientes}

El procedimiento de conglomerados jerárquico permite observar y deducir el número idóneo de clústeres con los que representar el comportamiento de los datos [37]. En el apartado anterior explicamos los departamentos o conglomerados fundidos en cada etapa y la distancia a la que se encontraban en el momento de la fusión. A partir de esta base, a continuación mostraremos los grupos resultantes, haciendo dos cortes en el proceso que definirán, en primer lugar, siete grupos de departamentos y, en segundo lugar, tres grupos. Consideramos estas dos agrupaciones como las más adecuadas para nuestro objetivo de describir el comportamiento de lealtad del paciente en los departamentos de salud de la Comunidad Valenciana.

\section{Resultado amplio: siete grupos de departamentos}

Hemos optado primeramente por una solución con siete conglomerados, fijando un primer corte en la etapa 17 (véase tabla 3) que define los siete grupos que mostramos a la izquierda de la línea gris del dendrograma ofrecido en la figura 3. 


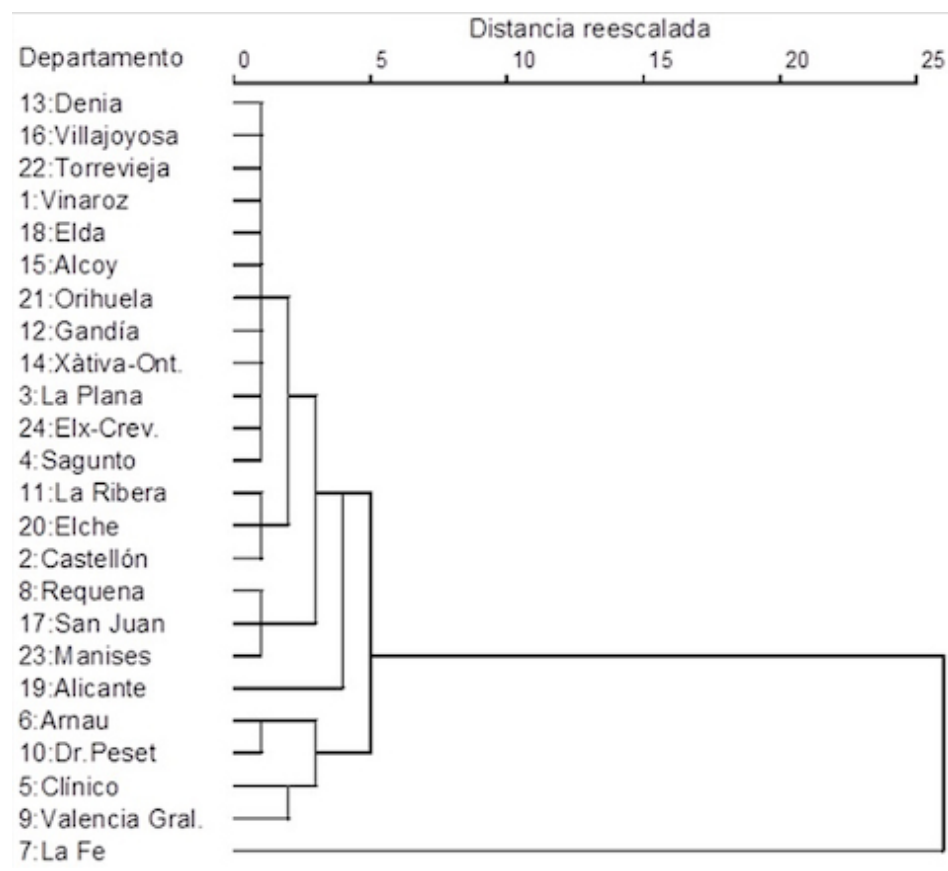

FIGURA 2

Dendrograma diferenciando siete grupos de departamentos Fuente: elaboración propia.

El dendrograma de la figura 2, además de representar las etapas de fusión, permite observar las distancias existentes entre los elementos, representadas mediante una escala estandarizada de veinticinco puntos. En primer lugar, apreciamos gráficamente la secuencia de integración de los departamentos, descrita en el anterior apartado. En segundo lugar, fijando el corte en la línea gris, encontramos siete grupos de departamentos con características homogéneas entre sí y heterogéneas entre ellos. Para una mejor visualización, en la figura 3 mostramos un diagrama de dispersión en el que se subrayan estos siete grupos y se aprecian sus características en cuanto a captación, retención y deserción de pacientes. 


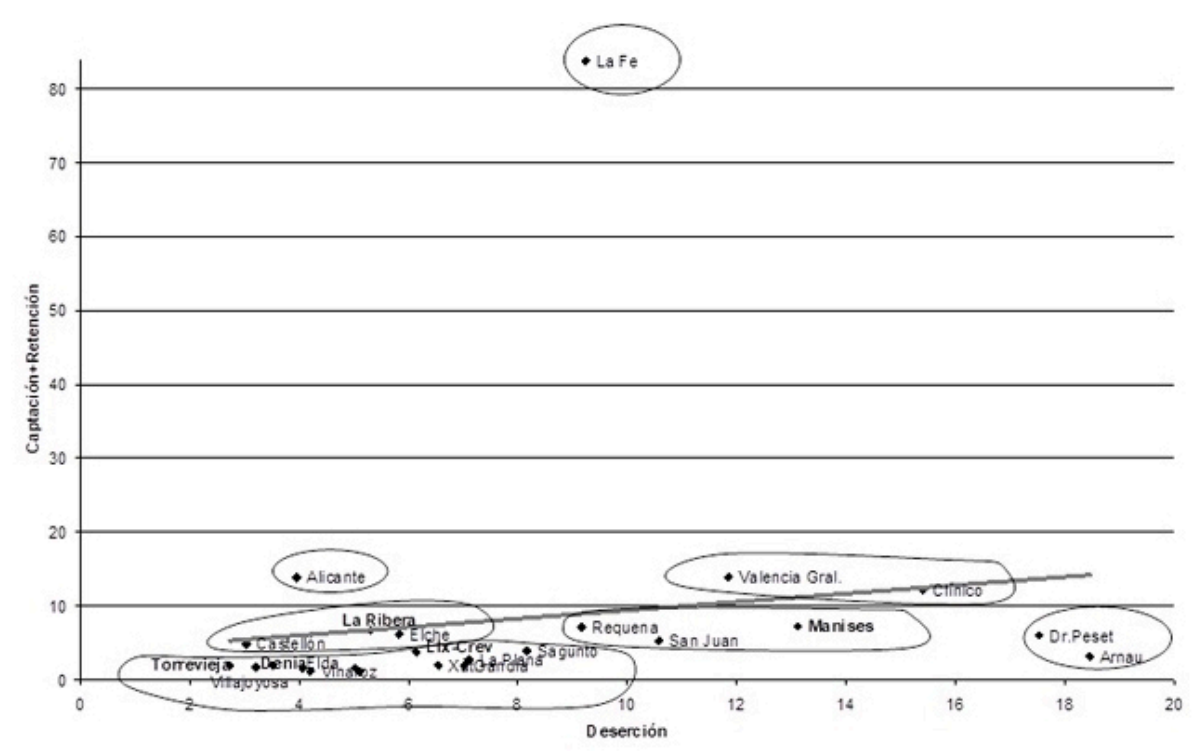

FIGURA 3

Diagrama de dispersión con siete grupos de departamentos

Fuente: elaboración propia.

Esta solución muestra un primer conglomerado formado por doce departamentos (véase tabla 3): Vinaroz, La Plana, Sagunto, Gandía, Denia, Xàtiva-Ontinyent, Alcoy, Villajoyosa, Elda, Orihuela, Torrevieja y ElxCrevillent. Se trata del grupo más numeroso, caracterizado por una escasa captación y retención de pacientes y una deserción de los pacientes asignados que no supera el 8\%. Un segundo conglomerado agrupa a Castellón, La Ribera y Elche. Estos tres departamentos muestran una captación y retención de pacientes ligeramente superior a la del primer conglomerado y una deserción en torno al 2.5\% - 6.0\%. El tercer grupo está formado por Requena, San Juan y Manises, con unas tasas de captación y retención similares al anterior grupo, pero con una mayor deserción de pacientes. El cuarto conglomerado integra a los departamentos Doctor Peset y Valencia-Arnau, que, manteniendo estas tasas de captación y retención, presentan los valores más elevados de deserción de pacientes. Valencia-Clínico y General de Valencia forman el quinto grupo: con una mayor captación y retención, que se sitúa en torno al 12\% y una deserción menor que el anterior grupo con valores comprendidos entre el 12 y el 16\%. Los dos últimos grupos cuentan con un solo departamento. Así, el sexto conglomerado corresponde al departamento de Alicante y el séptimo a La Fe. Se trata de dos departamentos con valores muy diferentes del resto. Alicante presenta una captación y retención del 14\% y apenas un 4\% de deserción de pacientes. Por su parte, La Fe, tal y como ya mostramos en análisis anteriores, cuenta con unos valores muy extremos: un $84 \%$ de captación y retención de pacientes frente a un $9 \%$ de deserción. 
TABLA 4

Conglomerado de pertenencia considerando siete grupos de departamentos

\begin{tabular}{|l|l|l|l|l|l|l|}
\hline \multicolumn{1}{|c|}{ Congl. 1 } & \multicolumn{1}{c|}{ Congl. 2 } & \multicolumn{1}{c|}{ Congl. 3 } & \multicolumn{1}{c|}{ Congl. 4 } & \multicolumn{1}{c|}{ Congl. 5 } & \multicolumn{1}{c|}{ Congl. 6 } & Congl. \\
\hline 1:Vinaroz & 2:Castellón & 8:Requena & 6:Arnau & 5:Clínico & 19:Alicante & 7:La Fe \\
3:La Plana & 11:La Ribera & 17:San Juan & 10:Dr. Peset & 9:Valencia Gral & & \\
4:Sagunto & 20:Elche & 23:Manises & & & & \\
12:Gandia & & & & & & \\
13:Denia & & & & & & \\
14:Xàtiva-Ont. & & & & & & \\
15:Alcoy & & & & & & \\
16:Villajoyosa & & & & & & \\
18:Elda & & & & & & \\
21:Orihuela & & & & & & \\
22: Torrevieja & & & & & & \\
24:Elx-Crev. & & & & & & \\
\hline
\end{tabular}

Fuente: elaboración propia.

En lo que respecta a nuestro objetivo secundario, relativo a los departamentos gestionados mediante el modelo Alzira, observamos cómo tres de ellos se encuentran en el conglomerado más numeroso, el primero, con tasas relativamente bajas de las tres variables: captación, retención y deserción de pacientes. Precisamente, Torrevieja se sitúa en el extremo inferior izquierdo al contar con la menor tasa de deserción y unas tasas reducidas de captación y retención. El departamento de La Ribera se encuentra ubicado en el segundo grupo, con una captación y retención de pacientes ligeramente superior y una deserción algo menor. Finalmente, Manises forma parte del tercer grupo, con unas tasas de captación y retención similares a La Ribera, pero una mayor deserción de pacientes.

La obtención de este resultado permite, desde un punto de vista de gestión, la segmentación de los departamentos de salud en función del comportamiento de lealtad observado en los pacientes. De este modo, se obtiene una segmentación como la plasmada en la figura 4, en la que se han coloreado las áreas geográficas de los departamentos según el conglomerado al que pertenecen. Esta segmentación aportará ventajas y facilidades de cara a la elaboración de políticas sanitarias y de determinación de la cartera de servicios. 


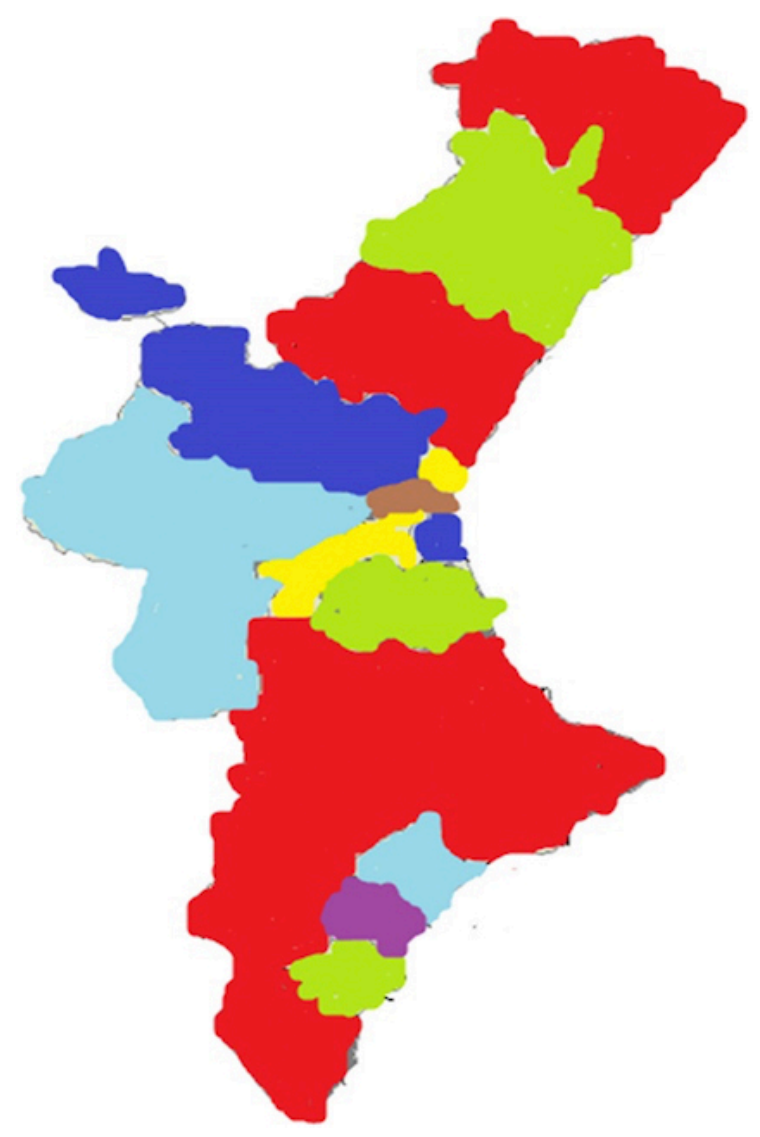

FIGURA 4

Mapa sanitario de la Comunidad Valenciana en función del comportamiento de lealtad observado en los pacientes (siete grupos)

Fuente: elaboración propia.

\section{Resultado estricto: tres grupos de departamentos}

En un segundo resultado, alejando el prisma de análisis, buscamos ofrecer una solución de tres grupos, con base en la distancia existente entre los casos y al modo en que se han ido formando los conglomerados. Para una mejor visualización, en el dendrograma de la figura 5 mostramos cómo hemos cortado el proceso de formación en la etapa 21 (véase tabla 3) dejando a la izquierda de la línea gris los tres grupos que describimos más adelante. 


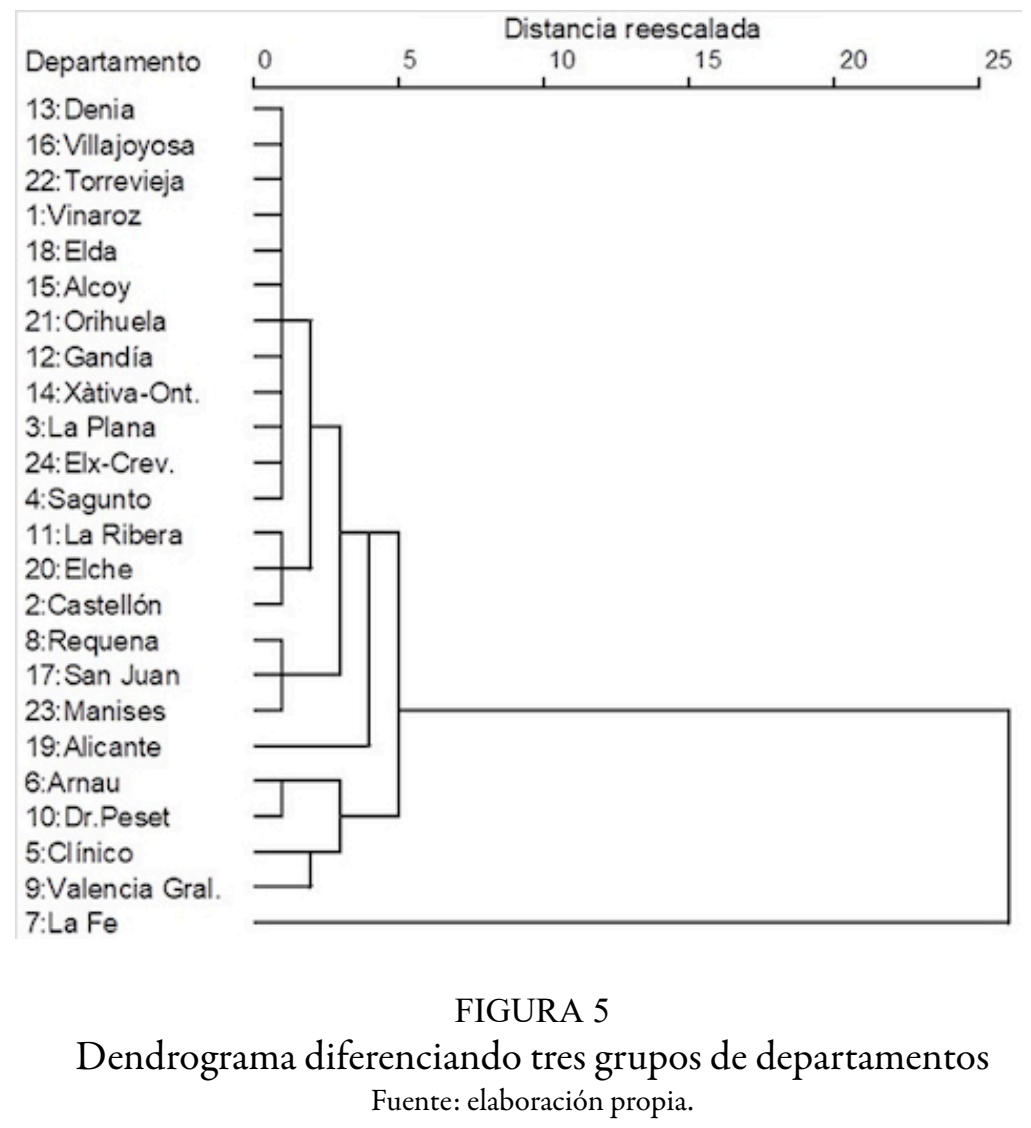

Tal y como muestra el dendrograma, los grupos uno, dos, tres y seis descritos en el anterior apartado se unen formando un nuevo conglomerado. Por otra parte, encontramos que los conglomerados cuatro y cinco se unen a su vez formando el segundo conglomerado. La Fe presenta valores muy aislados a los del resto de departamentos de salud de la Comunidad Valenciana. Por ello, al tratarse de un caso tan aislado, este departamento forma un grupo en sí mismo, no compartiendo sus características con las de ningún otro conglomerado. En esta visión, en la figura 6 presentamos un diagrama de dispersión en el que se encuentran agrupados estos tres conglomerados de departamentos. 


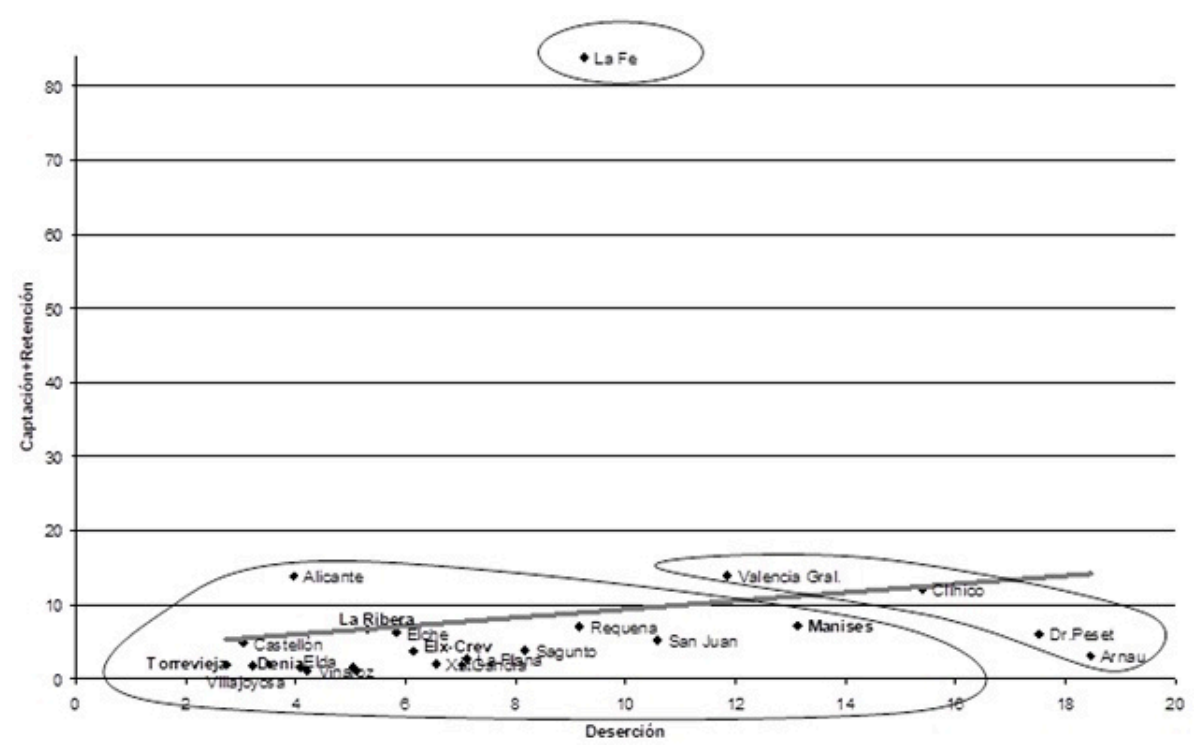

FIGURA 6

Diagrama de dispersión con tres grupos de departamentos

Fuente: elaboración propia.

El primer conglomerado, tal y como aparece señalado en el diagrama de dispersión, es el más numeroso, y está formado por los departamentos de Vinaroz, Castellón, La Plana, Sagunto, Requena, La Ribera, Gandía, Denia, Xàtiva-Ontinyent, Alcoy, Villajoyosa, San Juan, Elda, Alicante, Elche, Orihuela, Torrevieja, Manises y Elx-Crevillent. Este grupo se caracteriza por una captación y retención de pacientes entre 1\% y $14 \%$ y una deserción de los pacientes asignados que no supera el 14\%. En el segundo conglomerado se sitúan cuatro departamentos: Valencia-Clínico, Valencia-Arnau, General de Valencia y Doctor Peset. Aun teniendo una captación y retención de pacientes similar a la del primer conglomerado, su principal característica es su elevada deserción de pacientes. Finalmente, el tercer conglomerado contiene un único departamento: La $\mathrm{Fe}$, con una captación y retención que supera el 80\% y una deserción inferior al 10\%. Esta agrupación de departamentos en tres conglomerados queda recogida también en la tabla 5. 
TABLA 5

Conglomerado de pertenencia considerando tres grupos de departamentos

\begin{tabular}{l|l|l|}
\hline \multicolumn{1}{|c|}{ Congl. 1 } & \multicolumn{1}{c|}{ Congl. 2 } & \multicolumn{1}{c|}{ Congl. 3 } \\
\hline 1:Vinaroz & 5:Clinico & 7:La Fe \\
2:Castellón & 6:Arnau & \\
3:La Plana & 9:Valencia Gral & \\
4:Sagunto & 10:Dr. Peset & \\
8:Requena & & \\
11:La Ribera & & \\
12:Gandia & & \\
13:Denia & & \\
14:Xàtiva-Ont. & & \\
15:Alcoy & & \\
16:Villajoyosa & & \\
17:San Juan & & \\
18:Elda & & \\
19:Alicante & & \\
20:Elche & & \\
21:Orihuela & & \\
22:Torrevieja & & \\
23:Manises & & \\
24:Elx-Crev. & & \\
\hline
\end{tabular}

Fuente: elaboración propia.

En relación con los departamentos gestionados mediante el modelo Alzira (objetivo secundario del trabajo), esta solución de tres grupos engloba a las cinco concesiones en el primer conglomerado: con una captación, retención y deserción de pacientes que no supera el 14\%: ante una captación y retención similares a las del segundo conglomerado, observamos como principal característica de este grupo su menor deserción de pacientes asignados.

La obtención de este resultado en tres grupos permite, desde un punto de vista de gestión, la segmentación de los departamentos de salud en función del comportamiento de lealtad observado en los pacientes. De este modo, se obtiene la segmentación plasmada en la figura 7, en la que se han coloreado las áreas geográficas de los departamentos según el conglomerado al que pertenecen. 


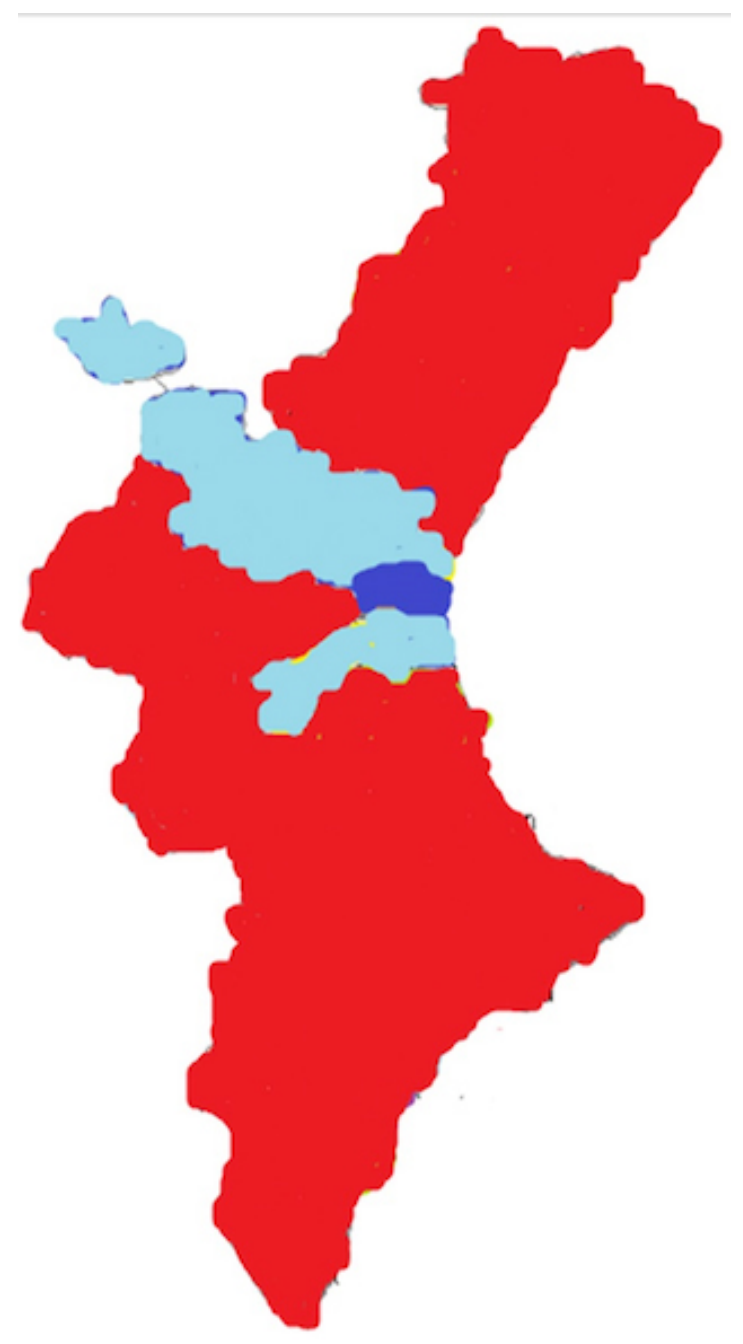

FIGURA 7

Mapa sanitario de la Comunidad Valenciana en función del comportamiento de lealtad observado en los pacientes (tres grupos) Fuente: elaboración propia.

A modo de resumen, en la tabla 4 ofrecemos los grupos de departamentos formados, distinguiendo entre siete y tres conglomerados, y sus características. En negrita subrayamos los gestionados mediante el modelo Alzira. 
TABLA 6

Grupos de departamentos formados y características principales

\begin{tabular}{|c|c|c|c|}
\hline \multicolumn{2}{|c|}{ Siete grupos } & \multicolumn{2}{|c|}{ Tres grupos } \\
\hline Departamentos & Características & Departamentos & Características \\
\hline $\begin{array}{l}\text { Vinaroz, La Plana, } \\
\text { Sagunto, Gandía, } \\
\text { Denia, Xàtiva- } \\
\text { Ontinyent, Alcoy, } \\
\text { Villajoyosa, Elda, } \\
\text { Orihuela, } \\
\text { Torrevieja, Elx- } \\
\text { Crevillent }\end{array}$ & $\begin{array}{l}\text { Escasa captación y } \\
\text { retención de } \\
\text { pacientes, y } \\
\text { deserción de } \\
\text { pacientes } \\
\text { asignados que no } \\
\text { supera el } 8 \%\end{array}$ & $\begin{array}{l}\text { Vinaroz, Castellón, } \\
\text { La Plana, Sagunto, } \\
\text { Requena, La } \\
\text { Ribera, Gandia, } \\
\text { Denia, Xàtiva- } \\
\text { Ontinyent, Alcoy, } \\
\text { Villajoyosa, San } \\
\text { Juan, Elda, } \\
\text { Alicante, Elche, } \\
\text { Orihuela, } \\
\text { Torrevieja, } \\
\text { Manises, Elx- } \\
\text { Crevillent }\end{array}$ & $\begin{array}{l}\text { Captación y } \\
\text { retención entre el } \\
1 \% \text { y el } 14 \%, y \\
\text { deserción de } \\
\text { pacientes } \\
\text { as ignados que no } \\
\text { supera el } 14 \%\end{array}$ \\
\hline $\begin{array}{l}\text { Castellón, La } \\
\text { Ribera, Elche }\end{array}$ & $\begin{array}{l}\text { Captación y } \\
\text { retención de } \\
\text { pacientes } \\
\text { ligeramente } \\
\text { superior a la del } \\
\text { primer grupo y } \\
\text { deserción en torno } \\
\text { al } 2,5 \%-6,0 \%\end{array}$ & & \\
\hline $\begin{array}{l}\text { Requena, San } \\
\text { Juan, Manises }\end{array}$ & $\begin{array}{l}\text { Tasas de } \\
\text { captación y } \\
\text { retención similares } \\
\text { al anterior grupo, } \\
\text { pero con mayor } \\
\text { deserción ( } 9 \%- \\
13 \%)\end{array}$ & & \\
\hline $\begin{array}{l}\text { Valencia-Arnau, } \\
\text { Doctor Peset }\end{array}$ & $\begin{array}{l}\text { Manteniendo estas } \\
\text { tasas de captación } \\
\text { y retención, } \\
\text { presentan los } \\
\text { valores más } \\
\text { elevados de } \\
\text { deserción }\end{array}$ & & \\
\hline $\begin{array}{l}\text { General de } \\
\text { Valencia, Valencia- } \\
\text { Clínico }\end{array}$ & $\begin{array}{l}\text { Mayor captación y } \\
\text { retención, en torno } \\
\text { al } 12 \% \text {, y deserción } \\
\text { entre el } 12 \text { y el } 16 \%\end{array}$ & $\begin{array}{l}\text { Valencia-Clínico, } \\
\text { Valencia-Arnau, } \\
\text { General de } \\
\text { Valencia, Doctor } \\
\text { Peset }\end{array}$ & $\begin{array}{l}\text { Captación y } \\
\text { retención de } \\
\text { pacientes similar a } \\
\text { la del primer grupo, } \\
\text { elevada deserción }\end{array}$ \\
\hline Alicante & $\begin{array}{l}\text { Captación y } \\
\text { retención del 14\% y } \\
\text { apenas un } 4 \% \text { de } \\
\text { deserción }\end{array}$ & & \\
\hline LaFe & $\begin{array}{l}\text { Captación y } \\
\text { retención que, de } \\
\text { manera conjunta, } \\
\text { superan el } 80 \% \text { y } \\
\text { una deserción } \\
\text { inferior al } 10 \%\end{array}$ & La Fe & $\begin{array}{l}\text { Captación y } \\
\text { retención que, de } \\
\text { manera conjunta, } \\
\text { superan el } 80 \% \text { y } \\
\text { una deserción } \\
\text { inferior al } 10 \%\end{array}$ \\
\hline
\end{tabular}

Fuente: elaboración propia. 


\section{Discusión}

\section{Interpretación y discusión de los resultados a la luz de las particularidades de la gestión sanitaria}

A partir de los resultados obtenidos y tratando de ofrecer una interpretación desde la perspectiva del comportamiento de lealtad del paciente, recordamos que la posición y formación de los diferentes conglomerados se ha realizado teniendo en cuenta, simultáneamente, las tasas de captación, retención y deserción de los pacientes que presentaban los diferentes departamentos. En esta visión, La Fe, que es el departamento que cuenta con el hospital más grande y más conocido de la Comunidad Valenciana, muestra un comportamiento de lealtad de los pacientes totalmente distinto al del resto de departamentos de salud. En segundo lugar, encontramos dos bloques de departamentos con una cierta homogeneidad en el comportamiento del paciente, pero heterogéneos entre ellos. Bajo este prisma y centrando nuestro interés en los departamentos gestionados mediante el modelo Alzira, encontramos que, si bien existen características en las que difieren, se sitúan en el conglomerado cuya característica diferenciadora es la menor deserción de los pacientes.

En primer lugar, se observa cómo estos resultados están en consonancia con otros estudios anteriores, como el realizado por Jiménez et al. [30] quienes, con el propósito de caracterizar y diferenciar los hospitales públicos de la Comunidad Valenciana según su producción de servicios y consumo de recursos, encuentran que La Fe constituye en sí mismo un grupo, alegando variables de tamaño, funcionamiento y actividad muy superiores a los hospitales de su tipo. En un segundo grupo, estos autores identifican hospitales de grandes dimensiones, pero con perfiles de actividad que no alcanzan a La Fe. Tal sería el caso del segundo grupo que identificamos en nuestro análisis y que está formado por los cuatro departamentos de la ciudad de Valencia, sin contar con La Fe.

En segundo lugar, extrapolándolo más allá del caso particular de la Comunidad Valenciana, de conformidad con el objetivo fijado para este trabajo, se han logrado aportaciones de índole metodológica y de gestión. En relación con la metodología, se ahonda en la utilidad del análisis clúster en el ámbito sanitario que, más allá de las aplicaciones encontradas en la literatura [29, 30, 31], se emplea en esta ocasión para el establecimiento de agrupaciones de áreas de salud con base en el comportamiento de lealtad de los pacientes. En un marco de división territorial del Sistema Sanitario de Salud, contar con metodología que permita el estudio de la oferta sanitaria y la respuesta del paciente como demandante del servicio sanitario resulta de especial utilidad para investigadores y gestores sanitarios. Desde una óptica de gestión, la agrupación en el comportamiento de lealtad del paciente permite, desde una visión macro, el análisis y adopción de políticas sanitarias a nivel de Comunidad Autónoma, clave para el futuro y la sostenibilidad de la sanidad pública española $[39,40]$, mientras que, desde una visión micro, favorece que los gestores de cada departamento puedan adaptar su oferta y servicio a las necesidades de los pacientes, en aras de lograr y mantener su lealtad (mayor captación y retención y menor deserción) [41].

Por otra parte, y en relación con el objetivo secundario planteado, el estudio realizado nos lleva a la conclusión de que los departamentos gestionados mediante colaboración público-privada (modelo Alzira) se caracterizan por una escasa deserción de pacientes (es decir, una vez que logran atender al paciente, este no abandona la relación). Se contribuye con esta conclusión a arrojar luz en el debate existente en la literatura sobre la utilidad e idoneidad de esta forma de gestión sanitaria en partenariado [42, 43, 44, 45, 46], al mostrar qué comportamiento real manifiesta el paciente cuando se le ofrece la posibilidad de elegir dónde desea ser atendido. Como se ha reflejado en este estudio, el paciente atendido en un departamento gestionado mediante 
colaboración público-privada opta por seguir acudiendo a este hospital antes que elegir una alternativa de gestión pública.

Al término de este trabajo, es justo reconocer limitaciones y proponer nuevas líneas de investigación para su subsanación. Cabe señalar como principal limitación la marcada territorialidad de los resultados, en tres sentidos. En primer lugar, el tamaño y repercusión de uno de los departamentos ( $\mathrm{La} \mathrm{Fe}$ ) ha marcado fuertemente la configuración de los conglomerados formados. En segundo lugar, no se conoce en qué medida la distancia a un hospital determinado ha podido afectar las decisiones de los pacientes; en futuros estudios cabría considerar esta variable. En tercer lugar, nuestro análisis de grupos se ajusta a la realidad de una región geográfica determinada. Su extrapolación a otros países es por tanto difícil. No obstante, existe la posibilidad de medición comparada de los flujos de pacientes entre departamentos en otras regiones, u otros países, lo que permitiría determinar el atractivo competitivo en materia de servicios sanitarios de unos departamentos respecto de otros. Además, el análisis efectuado podría completarse en futuros trabajos con otras variables explicativas del comportamiento, como las propias de los sujetos (edad, sexo o nacionalidad si se consideraran a los "turistas sanitarios") y del tipo de prestación (por especialidades, para evaluar la reagrupación de manera más afinada). En cualquier caso, el trabajo aquí expuesto ha evidenciado la bondad del análisis de grupos como guía para comprender mejor la idiosincrasia del servicio sanitario, e invita a futuras investigaciones en el ámbito de la lealtad de los pacientes, como una variable clave para la gestión sanitaria.

\section{Agradecimientos}

Artículo de investigación desarrollado a partir de un trabajo más amplio, concretamente una tesis doctoral titulada El comportamiento de lealtad del paciente: un análisis aplicado al Modelo Alzira, defendida por Remedios Calero en la Universidad Católica de Valencia (España), dirigida por la Dra. Martina G. Gallarza. Para el tratamiento estadístico del presente artículo ha colaborado Sergio Gadea.

\section{Referencias}

1. López Puig P, Alonso Carbonell L, García Milián AJ, Fernández Díaz IE. Presencia de los atributos para la integración de las redes de servicios de salud. Revista Cubana de Medicina General Integral. 2009; 25(4):86-97.

2. Álvarez Pérez AG, García Fariñas A, Rodríguez Salvá A, Bonet Gorbea M, de Vos P, Van der Stuyff P. La regionalización de los servicios de salud como una estrategia de reorganización sanitaria. Revista Cubana de Higiene y Epidemiología. 2008; 46(1).

3. Pérez Somalo F. Marketing en tecnología sanitaria. Madrid: ESIC Ediciones; 2008.

4. Jiménez M, Rivas JA, Zubia M. Un modelo de programación por metas para la elaboración del contrato-programa de un hospital público. Cuadernos de Gestión. 2008; 8(1):73-88.

5. Costa-Font J. Decentralization and the Spanish National Health System: soft budget constraint modernization. En Costa-Font J, Greer SL, editores. Federalism and decentralization in European health and social care. Londres: Palgrave Macmillan; 2013. p. 67-80.

6. España, Ley 14/1986, de 25 de abril, General de Sanidad.

7. Meneu R. Evaluación del buen gobierno sanitario. Algunas reflexiones para su mejora. En Ortún V, editor. El buen gobierno sanitario. Madrid: Springer Healthcare Communications; 2009. p. 11-26.

8. Fernández-Lasquetty J. Modelos de gestión en la Comunidad de Madrid: el modelo partenariado Público Privado. En Colomer J, Del Llano J, editores. Una nueva forma de trabajar... mirando al futuro. Madrid: Fundación Gaspar Casal; 2011. p. 123-126.

9. Calero R, Gallarza MG. Aplicabilidad del Service-Dominant Logic al ámbito sanitario: caracterizando el servicio para la co-creación de valor. Revista Gerencia y Políticas de Salud. 2015; (29):179-92. 
10. Vertinsky IB, Thompson WA, Uyeno D. Measuring consumer desire for participation in clinical decision making. Health Services Research. 1974; 9(2):121-34.

11. Gabbott M, Hogg G. Consumers and services. John Wiley and Sons; 1998.

12. Cram T. Estreche las relaciones con los clientes que cuentan. Prentice Hall International Editions; 2003.

13. Palmer A. Principles of services marketing. McGraw-Hill; 2005.

14. Mira JJ, Rodríguez-Marín J, Peset R, Ybarra J, Pérez-Jover V, Palazón I, Llorca E. Causas de satisfacción y de insatisfacción de los pacientes en hospitales y atención primaria. Revista de Calidad Asistencial. 2002; 17(5):273-83.

15. Molina-López M. Caja Castilla La Mancha: vinculando tecnologías e innovación sanitarias. En: Las claves de la sanidad futura: investigación y gestión; Ed. Funcas; 2008. pp. 116-20.

16. Zeithaml V, Bitner MJ, Gremler D. Services marketing. Integrating customer focus across the firm, sexta edición. Nueva York: McGraw-Hill International Edition; 2013.

17. Fornell C, Wernerfelt B. Defensive marketing strategy by customer complaint management: a theoretical analysis. Journal of Marketing Research. 1987; 24(4):337-46.

18. Rust RT, Zahorik AJ. Customer satisfaction, customer retention, and market share. Journal of Retailing. 1993; 69(2):193-14.

19. Johnson MD, Gustafsson A. Improving customer satisfaction, loyalty and profit: an integrated measurement and management system. Michigan: University of Michigan Business School Series; 2000.

20. Szwarc P. Researching customer satisfaction \& loyalty. Londres: Kogan Page; 2005.

21. Calero R, Gallarza MG. Loyalty behaviour in patients: applied analysis to the "Alzira Model”. International Review on Public and Nonprofit Marketing. 2014; 12(1):89-91.

22. Hair JF, Anderson RE, Tatham RL, Black WC. Análisis multivariante. Madrid: Prentice-Hall; 1999.

23. Vera JF, Rueda MM, González Carmona A, Arcos A, Miranda MDM, Galmiz ML. Estadística computacional en SPSS. Granada: autores; 2003.

24. Blankenship AB, Breen GE, Dutka A. State of the art Marketing Research. Chicago: American Marketing Association; 1998.

25. Churchill GA, Iacobucci D. Marketing research. Methodological foundations. Ohio: South-Western; 2002.

26. Grande I, Abascal E. Fundamentos y técnicas de investigación comercial. Madrid: ESIC Editorial; 2011.

27. Gebauer H. Identifying service strategies in product manufacturing companies by exploring environment-strategy configurations. Industrial Marketing Management. 2008; 37(3):278-91.

28. Natali PM. Análisis cluster: una aplicación a los departamentos de la provincia de Córdoba. Comunicación presentada en las XV Jornadas de intercambio de conocimientos científicos y técnicos. Río Cuarto, Argentina. 2008.

29. Jiménez WN, Barrachina I, Vivas D. Métodos multivariantes para la clasificación de hospitales: el caso de los hospitales públicos de la Comunidad Valenciana. Revista Gerencia y Políticas de Salud. 2004; 3(6):68-87.

30. López-Valcárcel BG, Barber P, Davila D. Clasificación de hospitales públicos españoles mediante el uso del análisis cluster. Informe técnico para el Ministerio de Sanidad, España, diciembre de 2003.

31. Alexander JA, Evashwick CJ, Rundall T. Hospitals and the provision of care to the aged: a cluster analysis. Inquiry. 1984; 21(4):303-12.

32. Base de datos COMPAS, registros año 2012. Autorización a su acceso por la Conselleria de Sanidad de la Generalitat Valenciana.

33. Bijnen EJ. Cluster analysis. The Netherlands: Tilburg University Press; 1973.

34. Sokal RR, Sneath PHA. Principles of numerical taxonomy. San Francisco: Freeman; 1963.

35. Malhotra NK, Birks DF. Marketing research. An applied approach. Harlow: Prentice Hall; 2003.

36. Veritt BS, Landau S, Leese M, Stahl D. Cluster analysis. Londres: Wiley; 2011.

37. Pardo A, Ruiz MA. SPSS 11. Guía para el análisis de datos. Madrid: McGraw-Hill; 2002. 
38. Bernal A. Técnicas de clasificación. En Martínez Gastey J et al, editor. La investigación en marketing. Barcelona: Aedemo; 2000. p. 603-08.

39. Repullo JR. Sistemas y servicios sanitarios. Madrid: Editorial Díaz de Santos; 2012.

40. Abellán JM. El sistema sanitario público en España y sus comunidades autónomas: sostenibilidad y reformas. Madrid: Fundación BBVA; 2013.

41. Ayuso D, Grande RF. La apertura de un hospital. Madrid: Editorial Díaz de Santos; 2012.

42. Oliva J, Ruiz-Giménez JL, Segura J. Qué hacemos por la sanidad pública. Madrid: Ediciones Akal; 2014.

43. Rubio M. Tu salud, nuestro negocio: Quién gana con el proceso de privatización de la Sanidad Pública en España. Madrid: Ediciones Akal; 2014.

44. Calero R, Gallarza MG. El modelo Alzira: Colaboración público-privada en gestión sanitaria: Un análisis de su especificidad. Madrid: Publicia; 2015.

45. Zaragoza G, Hernández-Aguado I, Parker LA. Colaboraciones público-privadas en salud pública: ¿parte de la solución o parte del problema? Gestión Clínica y Sanitaria. 2016; 18(3):75-7.

\section{Notas}

* Artículo de investigación.

\section{Licencia Creative Commons CC BY 4.0}

Como citar este artículo: Calero Gimeno R, Gallarza Granizo MG, Gadea Peña S. Segmentación y comportamiento de lealtad de los pacientes: un análisis clúster en los departamentos de salud de la Comunidad Valenciana. Rev Gerenc Polít Salud. 2017; 17(34): 1-23. https://doi.org/10.11144/ Javeriana .rgps 17-34.sclp 